The AstrophysicAl JouRnAL, 561:504-516, 2001 November 10

(C) 2001. The American Astronomical Society. All rights reserved. Printed in U.S.A.

\title{
POLARIZATION OF THE COSMIC MICROWAVE BACKGROUND FROM NONUNIFORM REIONIZATION

\author{
Guo-Chin Liu, ${ }^{1,2,3}$ Naoshi Sugiyama, ${ }^{2}$ Andrew J. Benson, ${ }^{4,5}$ C. G. Lacey, ${ }^{6}$ AND Adi Nusser ${ }^{7}$ \\ Received 2001 January 10; accepted 2001 July 11
}

\begin{abstract}
We study the polarization anisotropy in the cosmic microwave background (CMB) resulting from patchy reionization of the intergalactic medium by stars in galaxies. It is well known that the polarization of the CMB is very sensitive to the details of reionization, including the reionization epoch and the density fluctuations in the ionized gas. We calculate the effects of reionization by combining a semianalytic model of galaxy formation, which predicts the redshifts and luminosities of the ionizing sources, with a high-resolution $N$-body simulation, to predict the spatial distribution of the ionized gas. The models predict reionization at redshifts $z \sim 5-10$, with electron scattering optical depths due to reionization of $\sim 0.014-0.05$. We find that reionization generates a peak in the polarization spectrum with amplitude $\sim 0.05-0.15 \mu \mathrm{K}$ at large angular scales $(l \sim 3)$. The position of this peak reveals the size of the horizon at reionization, whilst its amplitude is a measure of the optical depth to reionization. On small scales $(l \gtrsim 6000)$, reionization produces a second-order polarization signal due to the coupling of fluctuations in the free electron density with the quadrupole moment of the temperature anisotropy. Careful study reveals that this coupling generates equal second-order polarization power spectra for the electric and magnetic modes, with amplitude $\sim 10 \mathrm{nK}$. This amplitude depends strongly on the total baryon density $\Omega_{b}$ and on the spatial correlations of the free electron density, and weakly on the fraction $f_{\text {esc }}$ of ionizing photons able to escape their source galaxy. The first- and second-order signals are therefore sensitive to different details of how the reionization occurred. Detection of these signals will place important constraints on the reionization history of the universe.
\end{abstract}

Subject headings: cosmic microwave background - cosmology: theory — galaxies: formation intergalactic medium

\section{INTRODUCTION}

The secondary anisotropies in the cosmic microwave background $(\mathrm{CMB})$ provide a laboratory for the study of the epoch of reionization in the universe. With the rapid improvement in $\mathrm{CMB}$ experimental sensitivity and resolution, it is timely to discuss the signals introduced by the process of reionization, which are at the $\mu \mathrm{K}$ level or below. In this paper, we concentrate on the CMB polarization in models with inhomogeneous reionization. It is well known that the primordial CMB polarization is generated at the recombination epoch through Thomson scattering of the quadrupole of the temperature anisotropy. The same mechanism operates at the reionization epoch and again distorts the shape of the polarization power spectrum. It has been pointed out by $\mathrm{Ng} \& \mathrm{Ng}$ (1996) and Zaldarriaga (1997) that this leads to the polarization anisotropy being suppressed on small scales but enhanced on large scales. The degree of suppression and enhancement typically depend on the optical depth to the recombination epoch produced by reionization. Although the amplitude of the polarization

\footnotetext{
${ }^{1}$ Department of Physics, Kyoto University, Kyoto 606-8502, Japan.

${ }^{2}$ Division of Theoretical Astrophysics, National Astronomical Observatory, Mitaka 181-8588, Japan.

${ }^{3}$ Institute of Astronomy and Astrophysics, Academia Sinica, Taipei 106, Taiwan.

${ }^{4}$ Astronomy Option, MC 105-24, California Institute of Technology, Pasadena, CA 91125.

${ }^{5}$ Department of Physics, Astronomy Section, University of Durham, Durham DH1 3LE, UK.

${ }^{6}$ Scuola Internazionale Superiore di Studi Avanzati, via Beirut 2-4, 34014 Trieste, Italy.

${ }^{7}$ The Physics Department, The Technion-Israel Institute of Technology, Technion City, Haifa 32000, Israel.
}

anisotropy is estimated to be much smaller than that in the temperature, it is advantageous to consider the polarization signal since it is generated when photons and electrons scatter for the last time. The temperature fluctuations are produced both by scattering between photons and electrons and by the gravitational redshift or blueshift caused by the evolution of the gravitational potential between the lastscattering epoch and the present (Sachs \& Wolfe 1967). On the other hand, the polarization is affected by the gravitational field only through gravitational lensing (Zaldarriaga \& Seljak 1998), which causes some smearing of the power spectrum and also some mixing of the "electric" and "magnetic" parity modes of the polarization. In Benson et al. (2001, hereafter Paper I), we presented a detailed calculation of inhomogeneous reionization of the intergalactic medium (IGM), using a semianalytic model of galaxy formation to predict the ionizing luminosities of stars in galaxies at different redshifts, and coupling this with an $\mathrm{N}$-body simulation to predict the spatial distribution of the ionizing sources and of the ionized gas. In this paper, we use the same model to predict the effects of reionization on the polarization of the CMB.

Reionization produces interesting effects on CMB temperature and polarization anisotropies at both first and second order in the perturbations. At first order, the effects of reionization are the same as for an IGM with spatially uniform density and ionized fraction. Density fluctuations in the free electrons around the reionization epoch produce CMB anisotropies only at second order, as in the Vishniac effect (Vishniac 1987). These second-order anisotropies are small in amplitude but nonetheless dominate over the firstorder anisotropies on small angular scales. They are thus cosmologically interesting as a probe of structure present at 
reionization. Historically, work on the second-order effects began by considering the temperature anisotropies in the case of a homogeneous reionization of the IGM, with the fluctuations in the free electron density being assumed to follow the variations in the total matter density, the socalled density modulation model (Vishniac 1987; Jaffe \& Kamionkowski 1998). However, in any realistic model, the reionization is expected to be patchy or inhomogeneous, with some regions already being fully ionized while others are still neutral, and the ionized regions growing until they encompass the whole IGM. Shapiro \& Giroux (1987) and Sato (1994) investigated the evolution of spherical ionization fronts in a homogeneous IGM using analytic methods. More recently, numerical simulations of radiative hydrodynamics have also shown how reionization proceeds in an inhomogeneous way (Gnedin 2000). Gruzinov \& Hu (1998) investigated second-order temperature anisotropies resulting from patchy reionization using a simple analytical model in which the universe is reionized in uncorrelated spherical patches. More realistic calculations that relax the assumption of uncorrelated spherical ionized regions have since been carried out by various authors, using either full simulations (Gnedin \& Jaffe 2001) or semianalytic methods (Paper I; Springel, White, \& Hernquist 2000; Bruscoli et al. 2000; Valageas, Balbi, \& Silk 2001). So far, these works have concluded that second-order effects make significant contributions to the temperature anisotropy on small scales $(l \gtrsim 3000)$, with peak amplitude in the range $0.1-1 \mu \mathrm{K}$.

While the second-order temperature anisotropies are well studied, those of the polarization have received relatively little attention. Seshadri \& Subramanian (1998) and Weller (1999) discussed this effect in density modulation and patchy reionization models, respectively. Here, we consider second-order polarization anisotropies in a more realistic model than has previously been possible. In our model, reionization results from photoionization by stars in galaxies, and the spatial fluctuations in the free electron density are the combined effect of fluctuations in the total density and fluctuations in the ionized fraction, thus combining the "density modulation" and "patchy reionization" effects. We calculate these effects in the same way as in our previous work (Paper I), by combining a semianalytic model of galaxy formation with an $\mathrm{N}$-body simulation of the distribution of dark matter in the universe to determine the distribution of ionized regions. In this paper, we consider only the scalar mode for the primordial fluctuations. In the case of first-order perturbation theory, the scalar mode fluctuations produce only the electric $(E-)$ mode of polarization (Zaldarriaga \& Seljak 1997). For the second-order perturbations, however, this may not be the case. Since the polarization anisotropies are produced by the coupling between the large-scale primordial CMB temperature quadrupole anisotropies and the small-scale density fluctuations in the ionized medium, axisymmetry is broken and the magnetic $(B$-) mode is also produced ( $\mathrm{Hu} 2000)$. In addition to the primordial CMB temperature quadrupole anisotropy, there is also a kinetic temperature quadrupole in the rest frame of the scatterers, produced by the quadratic Doppler effect from the peculiar velocity of the ionized medium (Sunyaev \& Zeldovich 1980). Coupling between this kinetic quadrupole and the electron density fluctuations can likewise produce a second-order contribution to the polarization, but this contribution is much smaller than the previous one (Hu 2000), so we neglect it in the present paper.
This paper is organized as follows. In $\S 2$, we present the Boltzmann equation for the first- and second-order polarization anisotropies. We then derive the $E$ - and $B$-mode power spectra by use of the Boltzmann equation. In $\S 3$, we give a brief overview of our model for inhomogeneous reionization and show our numerical results for both the first- and second-order polarization power spectra. Section 4 is devoted to our conclusions. Throughout this paper we work in units where $c=1$.

\section{SECOND-ORDER EFFECTS FROM THE REIONIZATION EPOCH}

\subsection{Boltzmann Equation and Second-Order Polarization}

The evolution of the temperature perturbation, $\Delta_{T}(x, \hat{n}, \tau)$, and the polarization perturbation, $\Delta_{P}(x, \hat{n}, \tau)$, are governed by the Boltzmann equation (Bond \& Efstathiou 1984; Peebles \& Yu 1970; Ma \& Bertschinger 1995). Here $\boldsymbol{x}$ is the comoving coordinate, $\tau \equiv \int d t / a$ the conformal time, where $a$ is the expansion factor normalized to unity today, and $\hat{n}$ the direction of photon propagation. Following Zaldarriaga \& Seljak (1997), to calculate the polarization perturbation $\Delta_{P}$ we work in terms of the perturbed Stokes parameters $\Delta_{Q \pm i U}($ see $\S 2.2$ ). In this paper, we do not include tensor perturbations in the metric, and we derive the Boltzmann equation in the conformal Newtonian gauge. Readers interested in the synchronous gauge or the transformation between these two gauges are referred to Ma \& Bertschinger (1995). In the Newtonian gauge, the perturbations are specified by two scalar potentials, $\phi$ and $\psi$, which play the role of the gravitational potential and the fractional perturbation to the spatial curvature, respectively. The Boltzmann equation then states that

$$
\begin{aligned}
\dot{\Delta}_{T}+\hat{n}_{i} \partial_{i} \Delta_{T} & =\dot{\phi}-\hat{n}_{i} \partial_{i} \psi+n_{e} \sigma_{T} a(\tau) \\
\times & {\left[-\Delta_{T}+\Delta_{T 0}+\hat{n}_{i} v_{b i}-\frac{\sqrt{5 \pi}}{5} \sum_{m=-2}^{m=2} Y_{2}^{m}(\hat{n}) \Pi^{(m)}\right], } \\
\Delta_{Q \pm i U}+\hat{n}_{i} \partial_{i} \Delta_{Q \pm i U}=n_{e} \sigma_{\mathrm{T}} a(\tau) & \\
\times & {\left[-\Delta_{Q \pm i U}+\sqrt{\frac{6 \pi}{5}} \sum_{m=-2}^{m=2} \pm 2 Y_{2}^{m}(\hat{n}) \Pi^{(m)}\right], }
\end{aligned}
$$

where ${ }_{s} Y_{l}^{m}$ is a spherical harmonic with spin weight $s$ and $\Pi^{(m)}$ is defined in terms of the quadrupole components of the temperature and polarization perturbations as

$$
\begin{aligned}
\Pi^{(m)}(x, \tau) \equiv & \Delta_{T 2}^{(m)}(x, \tau)+12 \sqrt{6} \Delta_{+, 2}^{(m)}(x, \tau) \\
& +12 \sqrt{6} \Delta_{-, 2}^{(m)}(x, \tau) .
\end{aligned}
$$

Here the derivatives are taken with respect to the conformal time, $v_{b}$ is the velocity of baryons, $n_{e}$ is the free electron number density, and $\sigma_{\mathrm{T}}$ is the Thomson cross section. We have also expanded the perturbations in the radiation field in spherical harmonics ${ }_{s} Y_{m}^{m}$ with appropriate spin weight $s$ (see also § 2.2)

$$
\begin{aligned}
\Delta_{T}(x, \hat{n}, \tau) & =\sum_{l m}(-i)^{l} \sqrt{4 \pi(2 l+1)} \Delta_{T l}^{(m)}(x, \tau) Y_{l}^{m}(\hat{n}), \\
\Delta_{Q \pm i U}(x, \hat{n}, \tau) & =\sum_{l m}(-i)^{l} \sqrt{4 \pi(2 l+1)} \Delta_{ \pm, l}^{(m)}(x, \tau)_{ \pm 2} Y_{l}^{m}(\hat{n}) .
\end{aligned}
$$

When we calculate the perturbations for a mode with wavenumber $\boldsymbol{k}$, we define the ${ }_{s} Y_{l}^{m}$ terms in a coordinate system 
for which the $z$-axis is parallel to $k$. To calculate the firstorder effect for scalar modes, we can set $m=0$, as in Zaldarriaga \& Seljak (1997), because of the axisymmetry of the radiation field around the mode axis for this case. However, their expansion is not valid for studying the second-order effect, in which the rotational symmetry around the wavevector is broken by coupling to other modes. It is very important to take into account $m \neq 0$ modes for the calculating the second-order effect, otherwise the (artificially imposed) axisymmetry guarantees that no magnetic mode can be generated. This is why Weller (1999), who assumed axial symmetry following Seshadri \& Subramanian (1998), obtained only the electric mode of polarization for the second-order effect. As was found by $\mathrm{Hu}$ (2000), who employed the weak-coupling approximation, the magnetic mode of polarization is also generated in the case of the second-order effect. Thus, the expansion in equation (4) is more general and useful for our calculation.

On small scales, as pointed out by Kaiser (1984), the contribution to secondary temperature anisotropies from homogeneous reionization tends to cancel along the line of sight. Thus, the first-order effect on the temperature and polarization anisotropies from the reionization epoch is suppressed at small angles. Hereafter, we concentrate on polarization and develop an equation for the visibilitymodulated effect that is the dominant source on small scales.

First, we write the inhomogeneous distribution of the free electron number density as

$$
n_{e}(\boldsymbol{x}, \tau)=\bar{n}_{e}(\tau)\left[1+\delta_{e}(\boldsymbol{x}, \tau)\right],
$$

where $\delta_{e}$ is the fluctuation in the electron number density and the overbar denotes the background quantity. Then, equation (2) can be rewritten in terms of Fourier modes as follows,

$$
\begin{aligned}
& \dot{\Delta}_{Q \pm i U}(\boldsymbol{k}, \tau)+i k \mu \Delta_{Q \pm i U}(\boldsymbol{k}, \tau) \\
& =\bar{n}_{e} \sigma_{T} a(\tau)\left\{-\Delta_{Q \pm i U}(\boldsymbol{k}, \tau)-R_{ \pm}(\boldsymbol{k}, \tau)+\sum_{m} \sqrt{\frac{6 \pi}{5}}{ }_{ \pm 2} Y_{2}^{m}(\hat{n})\right. \\
& \left.\times\left[\Pi^{(m)}(\boldsymbol{k}, \tau)+S^{(m)}(\boldsymbol{k}, \tau)\right]\right\}, \quad(6)
\end{aligned}
$$

where $S^{(m)}(k, \tau)$ and $R_{ \pm}(k, \tau)$ are the Fourier modes of the coupling of $\delta_{e}(x, \tau)$ to $\Pi^{(m)}(x, \tau)$ and $\Delta_{Q \pm i U}(x, \tau)$, respectively. In other words,

$$
\begin{aligned}
S^{m}(\boldsymbol{k}, \tau) & =\int d^{3} \boldsymbol{p} \delta_{e}(\boldsymbol{k}-\boldsymbol{p}, \tau) \Pi^{(m)}(\boldsymbol{p}, \tau) \\
\boldsymbol{R}_{ \pm}(\boldsymbol{k}, \tau) & =\int d^{3} \boldsymbol{p} \delta_{e}(\boldsymbol{k}-\boldsymbol{p}, \tau) \Delta_{Q \pm i U}(\boldsymbol{p}, \tau) .
\end{aligned}
$$

The polarization perturbations at the present time can be obtained by integrating the Boltzmann equation (6) along the line of sight (Zaldarriaga \& Seljak 1997)

$$
\begin{aligned}
& \Delta_{Q \pm i U}\left(\boldsymbol{k}, \mu, \tau_{0}\right)=\int_{0}^{\tau_{0}} d \tau e^{-i k\left(\tau_{0}-\tau\right) \mu} g(\tau) \\
& \quad \times\left\{\sqrt{\frac{6 \pi}{5}} \sum_{m} Y_{2}^{m}(\hat{n})\left[\Pi^{(m)}(\boldsymbol{k}, \tau)+S^{(m)}(\boldsymbol{k}, \tau)\right]-R_{ \pm}(\boldsymbol{k}, \tau)\right\},
\end{aligned}
$$

where $\mu=\boldsymbol{k} \cdot \hat{n}$, and $g(\tau)$ is the visibility function defined as

$$
g(\tau) \equiv-\frac{d \kappa}{d \tau} e^{\kappa\left(\tau_{0}\right)-\kappa(\tau)},
$$

with $\kappa(\tau) \equiv \int_{\tau}^{\tau_{0}} d \tau^{\prime} a n_{e} \sigma_{\mathrm{T}}$ being the electron-scattering optical depth. The visibility function has a simple physical meaning, being the probability that a photon had its last scattering at epoch $\tau$ and reaches the observer at the present time, $\tau_{0}$. Equation (9) can then be given a simple interpretation, since $\Pi^{(m)}(k, \tau)$ acts as a first-order source term, while $R_{ \pm}(k)$ and $S^{(m)}(k)$ are the contributions from the secondorder effect. To simplify the calculation, we neglect $R_{ \pm}(k)$ and $\Delta_{ \pm, 2}^{(m)}$ in $S^{(m)}(\boldsymbol{k})$ because the temperature perturbations typically dominate over the polarization perturbations. Furthermore, we assume the first-order temperature quadrupole $\Delta_{T 2}^{(m)}$ is uncorrelated with $\delta_{e}$ because the dominant contributions to these come from large and small scales, respectively. That is, we regard the source term for the second-order polarization as

$$
\begin{aligned}
S^{(m)}(\boldsymbol{k}, \tau) & \simeq \delta_{e}(\boldsymbol{k}, \tau) \int d^{3} \boldsymbol{p} \Delta_{T 2}^{(m)}(\boldsymbol{p}, \tau) \\
& \equiv \delta_{e}(\boldsymbol{k}, \tau) Q_{2}^{(m)}(\tau),
\end{aligned}
$$

with $Q_{2}^{(m)}(\tau)$ defined as $Q_{2}^{(m)}(\tau) \equiv \int d^{3} p \Delta_{T 2}^{(m)}(p, \tau)$ for convenience. Recall that the scalar mode in linear theory generates only the $m=0$ component in the $\boldsymbol{p}$-basis, i.e., $\Delta_{T 2}^{(0)} Y_{2}^{0}(\beta, \alpha)$, where $\beta$ and $\alpha$ are the polar and azimuthal angles defining $\hat{n}$ in this basis. Using the addition theorem, we can project the component in the $\boldsymbol{p}$-basis onto the $\boldsymbol{k}$ basis (see Ng \& Liu 1999),

$$
\sum_{m} Y_{l}^{m *}(\hat{n}) Y_{l}^{m}(\hat{p})=\sqrt{\frac{2 l+1}{4 \pi}} Y_{l}^{0}(\beta, \alpha) .
$$

It then follows that

$$
Q^{(m)}=\sqrt{\frac{4 \pi}{5}} \int d^{3} \boldsymbol{p} \Delta_{T 2}^{(0)}(\boldsymbol{p}) Y_{2}^{m *}(\hat{\boldsymbol{p}}) .
$$

Finally, the solution for $\Delta_{Q \pm i U}$ becomes

$$
\begin{aligned}
& \Delta_{Q \pm i U}\left(\boldsymbol{k}, \hat{n}, \tau_{0}\right)= \sqrt{\frac{6 \pi}{5}} \int_{0}^{\tau_{0}} d \tau e^{i k\left(\tau_{0}-\tau\right) \mu} g(\tau) \\
& \times \sum_{m} \pm 2 \\
& Y_{2}^{m}(\hat{n}) X^{(m)}(\boldsymbol{k}, \tau),
\end{aligned}
$$

where $X^{(m)}(\boldsymbol{k}, \tau)$ equals $\Pi^{(0)}(\boldsymbol{k}, \tau)$ or $S^{(m)}(\boldsymbol{k}, \tau)$ for the firstand second-order contributions, respectively.

\subsection{Stokes Parameters and the Power Spectrum}

We follow the approach of Zaldarriaga \& Seljak (1997) of calculating the polarization in terms of the electric and magnetic modes. We start with the description of the polarization perturbation in terms of the Stokes parameters $Q$ and $U$. If we consider a wave traveling in the $\hat{z}$ direction, $Q$ is the difference in intensity polarized in the $\hat{y}$ and $\hat{x}$ directions, while $U$ is the difference in the $(\hat{x}+\hat{y}) / \sqrt{2}$ and $(\hat{x}$ $-\hat{y}) / \sqrt{2}$ directions. The circular polarization parameter, $V$, cannot be produced by scattering, so we will not mention it further. Polarization is more complicated than temperature because the values for $Q$ and $U$ depend on the choice of coordinate system. Under a right-handed rotation through an angle $\phi$ around the $\hat{z}$-axis, the temperature is 
TABLE 1

$T_{(E, B) l}^{(m)}$ IN EQUATION (23)

\begin{tabular}{|c|c|c|c|}
\hline$m$ & \multicolumn{2}{|l|}{$T_{E l}^{(m)}$} & $T_{B l}^{(m)}$ \\
\hline $0 \ldots \ldots \ldots$ & \multicolumn{2}{|l|}{$(-i)^{l} \frac{j_{l}(k r)}{(k r)^{2}}$} & 0 \\
\hline $\pm 1 \ldots \ldots$ & \multicolumn{2}{|c|}{$\mp(-i)^{l} \frac{1}{(2 l+1) k r} \sqrt{\frac{1}{6 l(l+1)}}\left[l j_{l}(k r)-(l+1) j_{l-1}(k r)\right]$} & $\pm \sqrt{\frac{(-i)^{l}}{6 l(l+1)} \frac{j_{l}(k r)}{(k r)^{2}}}$ \\
\hline $\pm 2 \ldots \ldots$ & $\pm \frac{(-i)^{l}}{2 l+1} \sqrt{\frac{(l-2) !}{24(l+2) !}}\left\{\left[\frac{(l+2)(l+1)}{2 l-1}+\frac{l(l+1)}{2 l+3}+6 \frac{(2 l+1)(l-1)(l+2)}{(2 l-1)(2 l+3)}\right]\right.$ & $\left.\times j_{l}(k r)-(l+2)(l+1) \frac{j_{l-1}(k r)}{k r}+l(l-1) \frac{j_{l+1}(k r)}{k r}\right\}$ & $\mp \frac{(-i)^{l}}{2 l+1} \sqrt{\frac{(l-2) !}{6(l+2) !}}\left[(l+2) j_{l-1}(k r)-(l-1) j_{l+1}(k r)\right]$ \\
\hline
\end{tabular}


invariant, while $Q$ and $U$ transform according to

$$
(Q \pm i U)^{\prime}(\hat{n})=e^{\mp 2 i \phi}(Q \pm i U)(\hat{n}) .
$$

Thus the functions $Q \pm i U$ have spin \pm 2 and should be expanded in spherical harmonics of spin \pm 2 .

It is possible to produce a rotationally invariant measure of the polarization field if we introduce the spin raising and lowering operators $\partial$ and $\bar{\partial}$ (Newman \& Penrose 1966)

$$
\begin{aligned}
& ð \eta=-(\sin \theta)^{s}\left(\frac{\partial}{\partial \theta}+i \csc \theta \frac{\partial}{\partial \phi}\right)(\sin \theta)^{-s} \eta, \\
& \bar{\partial} \eta=-(\sin \theta)^{-s}\left(\frac{\partial}{\partial \theta}-i \csc \theta \frac{\partial}{\partial \phi}\right)(\sin \theta)^{s} \eta,
\end{aligned}
$$

with $\eta$ as a spin-s field. If they act on the spin-s spherical harmonics, we have

$$
\begin{aligned}
& \partial_{s} Y_{l m}=[(l-s)(l+s+1)]^{1 / 2}{ }_{s+1} Y_{l m}, \\
& \bar{\partial}_{s} Y_{l m}=-[(l+s)(l-s+1)]^{1 / 2}{ }_{s-1} Y_{l m} .
\end{aligned}
$$

The new bases of the rotationally invariant polarization field, called the electric mode and magnetic mode, are defined as

$$
\begin{aligned}
& \Delta_{E} \equiv-\frac{1}{2}\left(\bar{\partial}^{2} \Delta_{Q+i U}+\partial^{2} \Delta_{Q-i U}\right), \\
& \Delta_{B} \equiv-\frac{i}{2}\left(\bar{\partial}^{2} \Delta_{Q+i U}-\partial^{2} \Delta_{Q-i U}\right) .
\end{aligned}
$$

The values of $\Delta_{E}$ and $\Delta_{B}$ at a particular direction in the sky are independent of the coordinate system used to define them (unlike $\Delta_{Q}$ and $\Delta_{U}$ ). We work in the coordinate system where $\hat{k} \| \hat{z}$ and define $Q>0(Q<0)$ in the direction $\hat{e}_{\theta}\left(\hat{e}_{\phi}\right)$. For a scalar mode in first-order perturbation theory, the radiation field is axisymmetric around the wavevector. The polarization is produced by scattering of the quadrupolar component of the radiation field, and so it has only a $\Delta_{Q}$ component, but no $\Delta_{U}$ component (which would correspond to a polarization angle at $45^{\circ}$ to the $\hat{e}_{\theta}-\hat{e}_{\phi}$ axes), thus $\Delta_{Q+i U}=\Delta_{Q-i U}=\Delta_{Q}$. Furthermore, $\Delta_{Q}$ has no $\phi$-dependence, so $\bar{\partial}^{2}=\bar{\partial}^{2}$ (eq. [16]). As a consequence, $\Delta_{E}=$ $-ð^{2} \Delta_{Q}=-\bar{\partial}^{2} \Delta_{Q}$ and $\Delta_{B}=0$. This result is important because it shows that no magnetic mode can be produced by scalar modes (density perturbations) in first-order perturbation theory. However, as we will show later, the same situation does not occur in the second-order contribution to the polarization because the coupling between modes breaks axisymmetry.

Again, following Zaldarriaga \& Seljak (1997), the power spectra of the electric mode and magnetic mode can be defined as

$$
C_{(E, B) l} \equiv \frac{1}{2 l+1} \frac{(l-2) !}{(l+2) !} \sum_{m} \int d^{3} \boldsymbol{k}\left\langle\left|\Delta_{(E, B)}^{(m)}\left(\boldsymbol{k}, \tau=\tau_{0}\right)\right|^{2}\right\rangle,
$$

where $\Delta_{(E, B) l}^{(m)}$ can be extracted using

$$
\Delta_{(\boldsymbol{E}, \boldsymbol{B}) l}^{(m)}(\boldsymbol{k})=\int d \Omega Y_{l}^{m}(\theta, \phi) \Delta_{(\boldsymbol{E}, \boldsymbol{B})}(\boldsymbol{k}) .
$$

To calculate equation (20), we can apply the spin raising and lowering operators (eq. [16] or [17]) to the polarization perturbations in equation (14) twice. But the plane wave $e^{i k\left(\tau_{0}-\tau\right) \mu}$ itself carries an angular dependence; thus we expand the plane wave in a series of spherical waves using the Rayleigh equation

$$
e^{i k\left(\tau_{0}-\tau\right) \mu}=\sum_{l}(-i)^{l} \sqrt{4 \pi(2 l+1)} j_{l}\left[k\left(\tau_{0}-\tau\right)\right] Y_{l}^{0}(\hat{n}),
$$

where $j_{l}\left[k\left(\tau_{0}-\tau\right)\right]$ is the spherical Bessel function. Furthermore, we calculate the product of two spherical harmonics with spin by using the Clebsch-Gordan relation (Sakurai 1985)

$$
\begin{aligned}
s_{1} Y_{l_{1} s_{2}}^{m_{1}} Y_{l_{2}}^{m_{2}}= & \frac{\sqrt{\left(2 l_{1}+1\right)\left(2 l_{2}+1\right)}}{4 \pi} \\
& \times \sum_{l m s}\left\langle l_{1}, l_{2}, m_{1}, m_{2} \mid l, m\right\rangle \\
& \times\left\langle l_{1}, l_{2},-s_{1},-s_{2} \mid l,-s\right\rangle \\
& \times \sqrt{\frac{4 \pi}{2 l+1}}{ }_{s} Y_{l}^{m} .
\end{aligned}
$$

The expression for $\Delta_{(E, B) l}^{(m)}(k, \tau)$ then becomes

$$
\begin{aligned}
\Delta_{(E, B) l}^{(m)}(\boldsymbol{k}, \tau)= & \frac{3}{2} \sqrt{\pi} \frac{(l+2) !}{(l-2) !} \sqrt{2 l+1} \\
& \times \int_{0}^{\tau_{0}} d \tau g(\tau) X^{(m)}(\boldsymbol{k}, \tau) T_{(E, B) l}^{(m)}(k r),
\end{aligned}
$$

with $r=\tau_{0}-\tau$. In Table 1, we list the results of $T_{(E, B) l}^{(m)}(k r)$, in which the orthonormality relation

$$
\int d \Omega_{s} Y_{l}^{m *}{ }_{s} Y^{m^{\prime}}{ }_{l^{\prime}}=\delta_{l l^{\prime}} \delta_{m m^{\prime}}
$$

and the recursion relations of spherical Bessel functions

$$
\frac{j_{l}(x)}{x}=\frac{1}{2 l+1}\left[j_{l-1}(x)+j_{l+1}(x)\right]
$$

have been used. Finally, the power spectrum can be obtained by substituting equation (23) into (19),

$$
\begin{aligned}
C_{(E, B) l}= & (4 \pi)^{2} \frac{9}{16} \frac{(l+2) !}{(l-2) !} \sum_{m} \int k^{2} d k \\
& \times\left\langle\left|\int d \tau g(\tau) X^{(m)}(\boldsymbol{k}, \tau) T_{(E, B) l}^{(m)}(k r)\right| 2\right\rangle .
\end{aligned}
$$

In the case of the second-order effect, equation (26) can be rewritten as an expression involving the power spectrum of the fluctuations in the free electron density $\left\langle\delta_{e}(\boldsymbol{k}, \tau) \delta_{e}^{*}\left(\boldsymbol{k}, \tau^{\prime}\right)\right\rangle$, connecting the two different times $\tau$ and $\tau^{\prime}$. When we evaluate equation (26) numerically, we neglect the effect of the difference in times in the power spectrum, and so replace it by $\left\langle\delta_{e}(\boldsymbol{k}, \bar{\tau}) \delta_{e}^{*}(\boldsymbol{k}, \bar{\tau})\right\rangle$, evaluated at a single time $\bar{\tau}$ that is an average of $\tau$ and $\tau^{\prime}$. This should be an adequate approximation if the dominant wavelengths in equation (26) are small compared with the Hubble radius.

\section{NUMERICAL RESULTS}

To calculate the polarization anisotropy spectrum using the results discussed above, we use the publicly available code CMBFAST (Seljak \& Zaldarriaga 1996). We modify the ionization history in this code to follow the more realistic case from our previous work (Paper I), in which the reionization history of the universe is determined by a semianalytic model of galaxy formation. The semianalytic model 
is that of Cole et al. (2000), which includes the following processes: formatinon and merging of dark matter halos through hierarchical clustering; shock-heating and radiative cooling of gas within these halos; collapse of cooled gas to form galactic disks; star formation in disks and feedback from supernova explosions; galaxy mergers; chemical evolution of the stars and gas; and luminosity evolution of stellar populations based on stellar evolution codes and model stellar atmospheres. The model has been shown by Cole et al. (2000) to agree well with a wide range of observed properties of galaxies in the local universe. In Paper I we used this model to calculate the ionizing luminosities of galaxies at different redshifts, including the effects of absorption by interstellar gas and dust on the fraction of ionizing photons escaping, and followed the propagation of the ionization fronts around each galaxy. To find the ionizing luminosity, we first calculate the rate at which ionizing photons are being produced by stars in the galaxy, then apply an attenuation due to dust, and finally allow a fraction $f_{\text {esc }}$ of the remaining photons to escape into the IGM. The mass of ionized hydrogen in each spherical ionization front is found by integrating the equation (Shapiro \& Giroux 1987)

$$
\frac{1}{m_{\mathrm{H}}} \frac{d M}{d t}=S(t)-\alpha_{\mathrm{H}}^{(2)} a^{-3} f_{\text {clump }} n_{\mathrm{H}} \frac{M}{m_{\mathrm{H}}},
$$

where $n_{\mathrm{H}}$ is the comoving mean number density of hydrogen atoms (total, $\mathrm{H} \mathrm{I}$, and $\mathrm{H} \mathrm{II}$ ) in the IGM, $m_{\mathrm{H}}$ is the mass of a hydrogen atom, $a(t)$ is the scale factor of the universe normalized to unity at $z=0, t$ is time (related to the conformal time by $d t=d \tau / a), S(t)$ is the rate at which ionizing photons are being emitted, and $\alpha_{\mathrm{H}}^{(2)}$ is the case $\mathrm{B}$ recombination coefficient. The clumping factor $f_{\text {clump }} \equiv\left\langle\rho_{\mathrm{IGM}}^{2}\right\rangle / \bar{\rho}_{\mathrm{IGM}}^{2}$ gives the effect of clumping on the recombination rate of hydrogen in the IGM. A larger $f_{\text {clump }}$ increases the recombination rate resulting in a delay of the reionization epoch. We use the clumping factor $f_{\text {clump }}^{\text {(halos) }}$ as defined in Paper I. By summing over the ionized volumes due to all galaxies, we can calculate the fraction of the IGM that has been reionized at any redshift. Having this reionization history, we can then obtain the first-order power spectrum of the polarization anisotropies without making any assumptions about the spatial distribution of the ionized gas. We consider only the scalar mode of the primordial perturbations, for which the radiation field for each $\boldsymbol{k}$-mode is axially symmetric. Thus, we set $m=0$ in equation (26) and find no magnetic mode can be produced (see Table 1).

The second-order effect is more complicated, as the source term contains $\delta_{e}$ and $\Delta_{T 2}^{(m)}$, i.e., the $m=0, \pm 1$ and \pm 2 components of the temperature quadrupole must be considered. The time evolution of the temperature quadrupole components with different $m$ in the $\boldsymbol{k}$-basis is obtained using equation (13), and $\Delta_{T 2}^{(0)}$ is calculated using the modified CMBFAST code. This leaves only the power spectrum of the electron density fluctuations $\delta_{e}$ unknown, which should be calculated from the distribution of ionized regions with different sizes and shapes and with a correlated spatial distribution. An exact calculation would require a high-resolution numerical simulation with gasdynamics and radiative transfer included, and so would require very large amounts of computing time. Here we instead calculate the density field $\delta_{e}$ of the ionized gas using the simpler approach of Paper I, in which the semianalytic galaxy for- mation model is combined with a high-resolution $N$-body simulation of the dark matter. The simulation volume, which is a box of comoving length $141.3 h^{-1} \mathrm{Mpc}$ and contains $256^{3}$ dark matter particles each of mass $1.4 \times 10^{10}$ $h^{-1} M_{\odot}$, is divided into $256^{3}$ cubical cells of comoving size $0.55 h^{-1}$ Mpc. Each dark matter halo in the simulation containing more than 10 particles is populated with galaxies according to the semianalytic model, and the sum of their ionizing luminosities placed in a source at the center of mass of the halo. Ionizing photons that originate from galaxies in lower mass halos (which are not resolved in the simulation) are added in by assuming that these unresolved halos trace the remaining mass of the simulation (i.e., the mass that is not part of a resolved halo). In Paper I we demonstrated that the locations of unresolved sources do not significantly affect the resulting anisotropy spectrum. We determine which regions of the simulation box become ionized by using one of the five toy models A-E listed below, which span the likely range of possible behavior. In all cases, the total mass of ionized hydrogen in the simulation box is the same and is forced to equal that for a large-scale homogeneous distribution with the specified IGM clumping factor $f_{\text {clump }}$, calculated by use of equation (27). We assume that the total gas density in the IGM traces the dark matter, thus neglecting the effects of pressure in the IGM. $\mathrm{Hu}(2000)$ has shown that this is a good approximation for anisotropies with $l \lesssim 10^{4}$.

Model A (Growing front model).--Ionize a spherical volume around each halo with a radius equal to the ionization front radius for that halo calculated assuming a largescale uniform IGM. Since in the simulation the IGM is not uniform but is assumed to trace the dark matter, and also because some spheres will overlap, the ionized volume calculated in this way will not contain the correct total ionized mass. We therefore scale the radius of each sphere by a constant factor, $f$, and repeat the procedure. This process is repeated, with a new value of $f$ each time, until the correct total mass of hydrogen has been ionized.

Model B (High-density model).- - In this model, we ignore the positions of halos in the simulation. Instead, we simply rank the cells in the simulation volume by their density. We then completely ionize the gas in the densest cell. If this has not ionized enough $\mathrm{H}$ I then we ionize the second densest cell. This process is repeated until the correct total mass of $\mathrm{H} \mathrm{I}$ has been ionized.

Model C (Low-density model).-This is like model B, except that we begin by ionizing the least dense cell, and work our way up to cells of greater and greater density. This model mimics that of Miralda-Escudé, Haehnelt, \& Rees (2000).

Model D (Random spheres model).-As for model A, except that the ionized spheres are placed at completely random positions in the simulation volume, rather than on the dark matter halos to which they belong. By comparing with model $\mathrm{A}$, this model allows us to estimate the importance of the spatial clustering of dark matter halos.

Model E (Boundary model).--Ionize a spherical region around each halo with a radius equal to the ionization front radius for that halo. This may ionize too much or not enough $\mathrm{H}$ I depending on the density of gas around each source. We therefore begin adding or removing cells at random from the boundaries of the already ionized regions until the required mass of $\mathrm{H} \mathrm{I}$ is ionized. 
From the results of the simulation, we calculate $\delta_{e}$ as

$$
\delta_{e}(\boldsymbol{x}, \tau)=\frac{x_{e}(\boldsymbol{x}, \tau)(1+\delta)}{\bar{x}_{e}(\tau)}-1,
$$

where $x_{e}$ is the ionized fraction (which we take to equal 1 in ionized regions and 0 in neutral regions) and $\delta$ is the dark matter overdensity (i.e., we assume that fluctuations in the gas density follow those in the dark matter). Here $\bar{x}_{e}$ is defined as the mass-averaged ionized fraction in the IGM.

We fix the cosmological parameters to be $\Omega_{0}=0.3, \Lambda_{0}=$ 0.7 , Hubble constant $H_{0}=70 \mathrm{~km}^{-1} \mathrm{~s}^{-1} \mathrm{Mpc}^{-1}$ and $\sigma_{8}=$ 0.9 . We will consider the effects of varying $f_{\text {esc }}$ and $\Omega_{b}$ on the polarization anisotropies and also examine the polarization power spectrum in all five toy models for the distribution of ionized regions. In Figure 1 we plot the visibility functions of the ionization histories in our simulation for different $f_{\text {esc }}$ (Fig. $1 a$ ) and different $\Omega_{b}$ (Fig. $1 b$ ). We find that the visibility function depends strongly on $f_{\text {esc }}$, and also on $\Omega_{b}$. The value of $\Omega_{b}$ determines the cooling rate (and so star formation rate) in our model of galaxy formation and also alters the recombination rate in the IGM, and so affects the time at which reionization occurs (Fig. 1b). Note that when we vary $\Omega_{b}$ and $f_{\text {esc }}$, we also vary the fraction of brown dwarfs in the initial mass function used in the galaxy formation model, in such a way that the model always fits the "knee" of the observed $\mathrm{H} \alpha$ luminosity function of galaxies at $z=0$ (see Paper I for more details). Therefore the production of ionizing photons does not simply scale with $\Omega_{b}$.

In Figure 2, we show how the first-order polarization anisotropies are affected by scattering by free electrons at the reionization epoch, calculated using the modified CMBFAST code. We find, first, that rescattering at the reionization epoch generates a new anisotropy at large scales because the horizon has grown to a much larger size by reionization than it had at recombination $(z \simeq 1100)$.
More specifically, the location of the new peak reveals the horizon size at last scattering, and its height reveals the duration of last scattering, i.e., this new peak is sensitive to the optical depth produced by reionization. To see how the distortion of the primordial polarization depends on the optical depth $\kappa_{i}$ back to the start of reionization, we plot the resulting power spectrum for various values of $f_{\text {esc }}$ in Figure $2 a$. We find that the boost in the large-scale power depends strongly on the value of $f_{\text {esc }}$. (The optical depth is 0.034 , 0.017 , and 0.014 for $f_{\text {esc }}=1.0,0.1$, and 0.05 , respectively, for $\Omega_{b}=0.02$.) The location of the new peak depends on the reionization epoch, which is characterized by the redshift $z_{i}$ of the peak of the visibility function as shown in Figure 1. From numerical simulations of the Boltzmann code for different reionization epochs and cosmological parameters, we obtain a fitting formula for the peak location $l_{\text {peak }}$ as a function of the reionization epoch $z_{i}$ :

$$
l_{\text {peak }}=0.74\left(1+z_{i}\right)^{0.73} \Omega_{0}^{0.11} .
$$

This fit is consistent with the peak locations shown in Figure 2. On the other hand, the first-order polarization fluctuations are suppressed on small scales by rescattering since a fraction of the photons coming from the recombination epoch is scattered after reionization. The tiny optical depth for rescattering causes little erasure of power on small scales, but the new peak reaches an amplitude of $\sim 0.05-0.1$ $\mu \mathrm{K}$. From Figure $2 b$ we can see that the suppression also depends on $\kappa_{i}$.

Let us discuss now the second-order effect. In Figure 3, we show the contribution to $C_{E l}$ and $C_{B l}$ from each value of $m$ for model A. For $m=0$, only the $E$-mode is generated because $T_{B l}^{(0)}=0$ (see Table 1). Our numerical results show that the $E$-mode (the $B$-mode) from $m= \pm 2(m= \pm 1)$ dominates the one from $\pm 1( \pm 2)$ by more than 4 orders of magnitude. However, the total power spectra of the $E$ - and

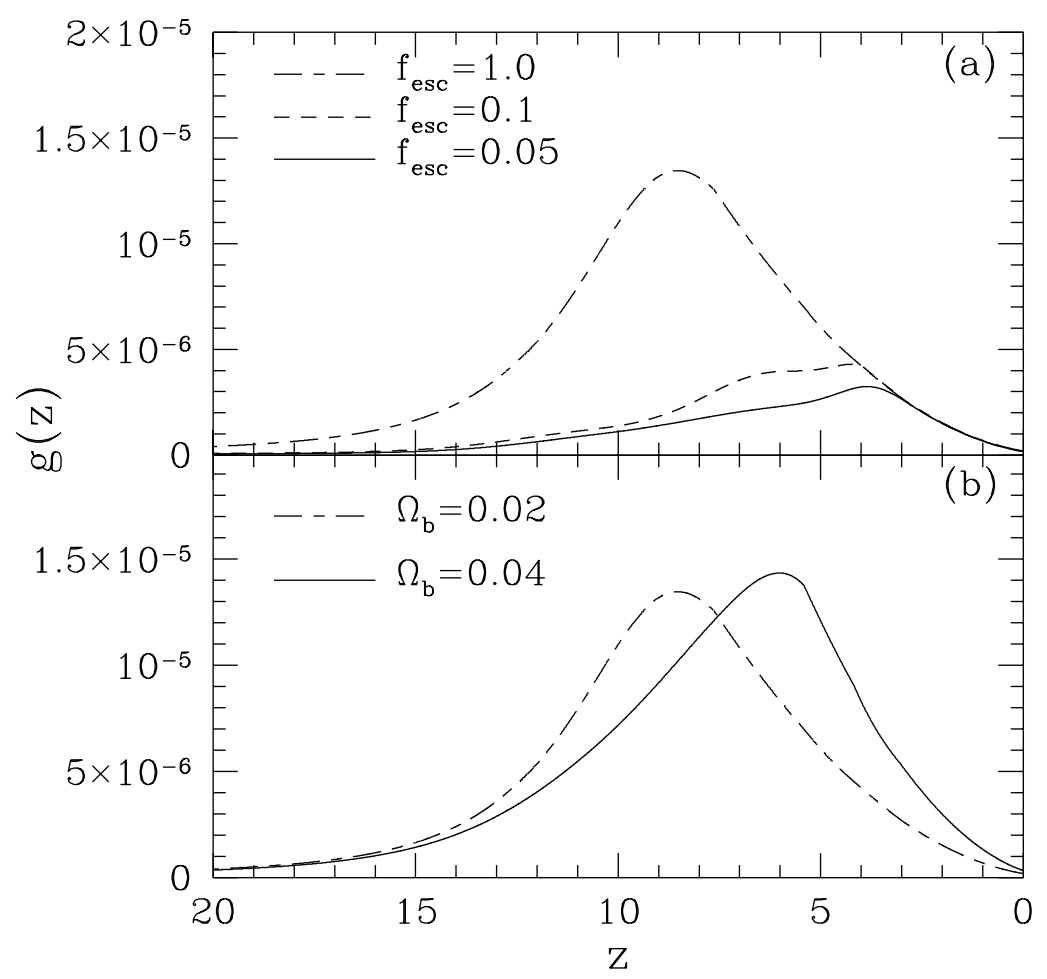

FIG. 1.-Visibility function for various $(a)$ escape fractions $f_{\text {esc }}$ and $(b)$ baryon fractions $\Omega_{b}$ in $\Lambda$ CDM models with $\Omega_{0}=0.3, h=0.7$, and $\Lambda=0.7$. In $(a)$, $\Omega_{b}$ is fixed at 0.02 , and in $(b), f_{\text {esc }}$ is set to 1.0 . 


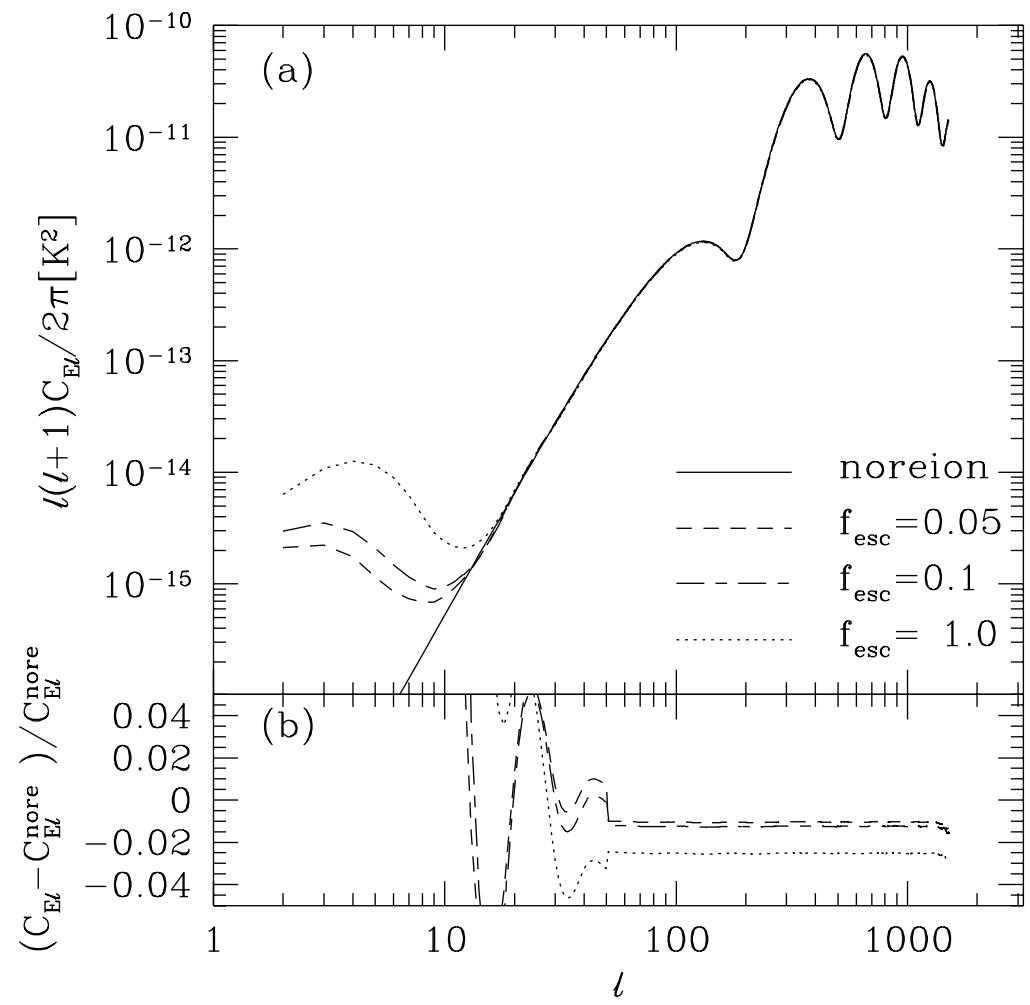

Fig. 2.-Modification of the first-order polarization power spectrum by reionization. (a) The angular power spectrum of the first-order polarization produced by the reionization histories with $\Omega_{b}=0.02$ and different escape fractions $f_{\text {esc }}$. $(b)$ The fractional change in these power spectra relative to the model with no reionization. The boost on large scales and supression on small scales are due to reionization. The values of $f_{\text {esc }}=0.05,0.1$, and 1.0 correspond to optical depths due to reionization of $\kappa_{i}=0.014,0.017$, and 0.034 , respectively, for $\Omega_{b}=0.02$. In panel $a$, the power spectrum is multiplied by the square of CMB temperature $T_{\mathrm{CMB}}=2.726 \mathrm{~K}$.

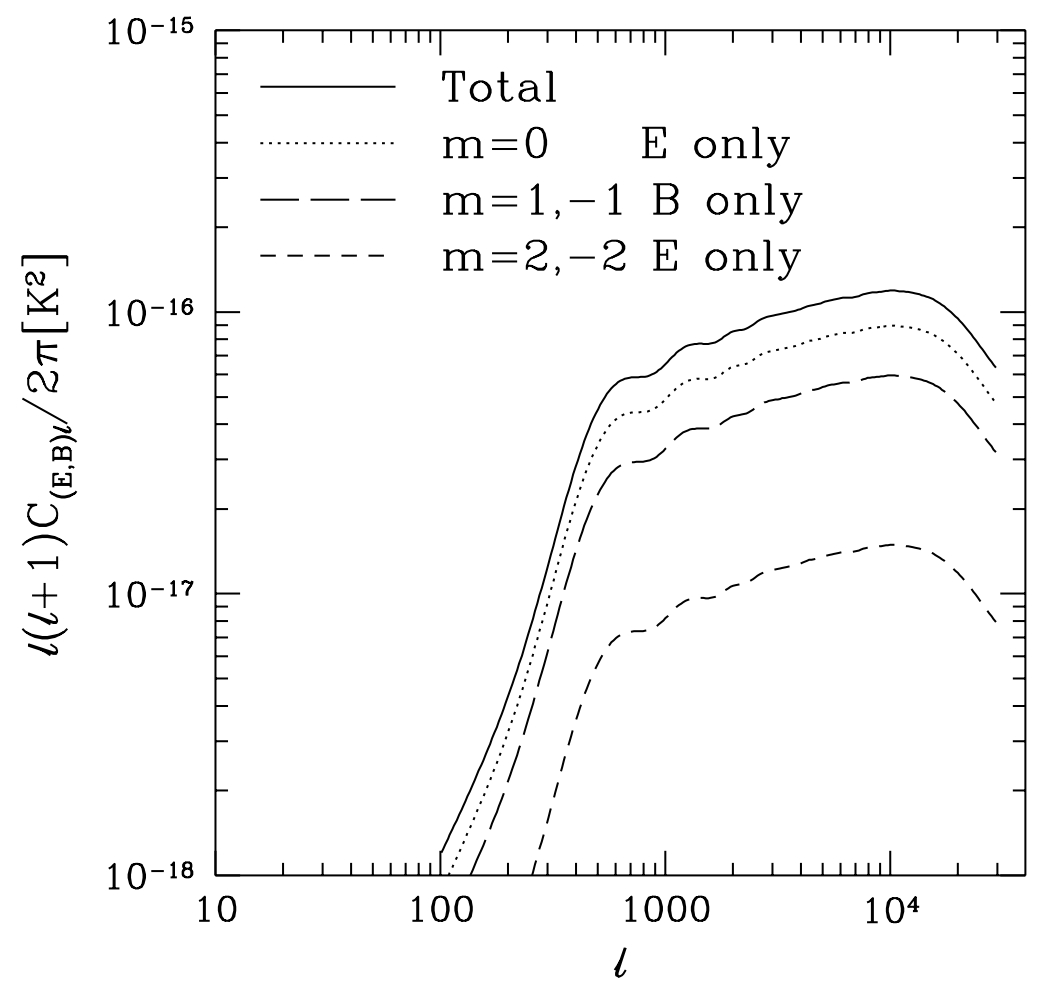

FIG. 3. - Contributions to the angular power spectrum of polarization from the second-order effect from different values of $m$. The curves shown are computed using model A with $f_{\text {esc }}=1$. Note that the long-dashed curve shows the contribution to the $B$-mode from only $m=1$ or $m=-1$ (not the sum of $m=1$ and $m=-1$ ), and similarly the short-dashed curve shows the contribution to the $E$-mode from only $m=2$ or $m=-2$. We find that the dominant contribution to the $E$-mode is from $m=0$ and $m= \pm 2$, while for the $B$-mode it is from $m= \pm 1$. There is no $B$-mode for $m=0$ and very small $E$ - and $B$-modes for $m= \pm 1$ and $m= \pm 2$, respectively. The total power spectrum of the $E$-mode, plotted as the solid curve, and calculated by summing the $m=0, \pm 1$ and \pm 2 contributions, is the same as the total power spectrum of the $B$-mode. The power spectra are multiplied by $T_{\mathrm{CMB}}^{2}$. 
$B$-modes are almost exactly the same. The difference is less than $10^{-6}$, which may be caused by numerical errors. These results are consistent with previous work by $\mathrm{Hu}(2000)$, in which the weak-coupling approximation was employed. (The weak-coupling approximation involves making an analytical approximation for the line-of-sight integrals, while in equation (26) we instead calculate the integrals over $\tau$ and $k$ numerically.) The reason for the equality of the total $E$ - and $B$-mode power spectra is essentially that the firstorder quadrupole whose scattering produces the polarization is dominated by large scales, and so has a random orientation relative to the small-scale fluctuations in the electron density (see eq. [11]). Scattering of the quadrupole by the small-scale fluctuations therefore on average excites $E$ - and $B$-modes equally.

Numerically, the amplitude of the second-order signal is found to be about $10^{-8} \mathrm{~K}$. This can also be understood as follows from (eq. [14]), the amplitude of the secondorder polarization anisotropies is roughly $\Delta_{Q \pm i U} \sim$ $\int d \tau g(\tau) Q_{2}^{(m)} \delta_{e} \sim \kappa Q_{2}^{(m)} \delta_{e}$. In our calculation $\kappa, Q_{2}^{(m)}$ and $\delta_{e}$ are typically on the order of $10^{-2}, 10^{-5}$, and $10^{-1} \mathrm{~K}$, respectively. The order of magnitude of the second-order contribution then follows.

The relation between the angular wavenumber $l$ and the comoving wavenumber $k$ of the density fluctuations at a given redshift is

$$
l \sim k r(z)=k \int_{0}^{z} \frac{d z}{H_{0} \sqrt{\Omega_{0}(1+z)^{3}+1-\Omega_{0}}},
$$

where $r(z)$ is the standard radial coordinate distance in the Robertson-Walker metric, and the final expression assumes a flat cosmology. For our standard choice $\Omega_{0}=0.3, \Lambda_{0}=$
0.7 , we find $r(z) \approx 6000 h^{-1} \mathrm{Mpc}$ for reionization at $z_{i} \sim$ 5-10. Thus, electron density fluctuations at reionization on comoving scale $\Delta x$ produce second-order anisotropies at $l \sim 6000\left(h^{-1} \mathrm{Mpc} / \Delta x\right)$.

In Figure 4, we plot the second-order power spectrum of the polarization in the five toy models with fixed extreme escape fraction $f_{\text {esc }}=1$ and $\Omega_{b}=0.02$. Although the shapes of the curves are all very similar, their amplitudes are different. Note that the reduction in power above $l \sim 10,000$ is artificial and due to the limited resolution of the $N$-body simulation we use (the density field of the ionized gas is calculated on a grid with cell size $0.55 h^{-1} \mathrm{Mpc}$, corresponding to $l \sim 10^{4}$ ). On the other hand, the finite size of the simulation box $\left(256 h^{-1} \mathrm{Mpc}\right)$ affects the power spectrum for $l$ below a few hundred. We see that the amplitude of the power spectrum around the peak $(l \approx 10,000)$ varies by a factor $\approx 2.5$, depending on which of the models $\mathrm{A}-\mathrm{E}$ is used. In Paper I we argued that the amplitude of the curves is affected by the strength of the correlations of $\delta_{e}$ present in each model. As a result, the "high-density" model B is the most strongly correlated and has the highest amplitude, and conversely the "low-density" model $\mathrm{C}$ has the lowest amplitude.

In Figure 4, we also compare our results to the analytical toy model of Gruzinov \& $\mathrm{Hu}$ (1998), in which the reionization is described by three free parameters. In their model, each luminous source is assumed to ionize a spherical region with fixed comoving radius $R$, the first source appears at redshift $z_{i}$, and new sources turn on at a constant rate until reionization is complete after an interval $\delta z$. An artificial assumption is made that luminous sources appear at random locations in space, so there are no correlations between the positions of the ionized spheres. Assuming that

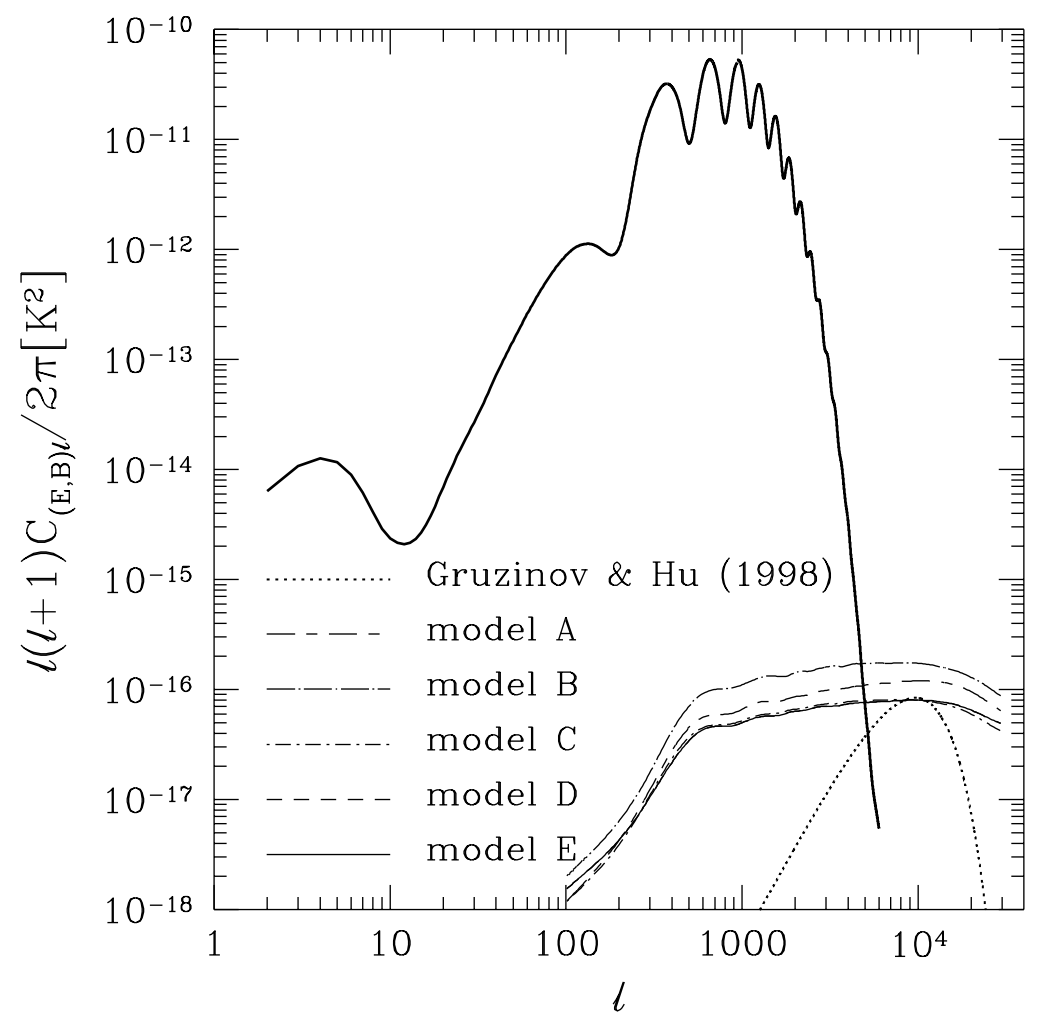

FIG. 4.- Power spectra of the second-order effect for our models (note that the curve for model D is hidden under that for model E). For comparison, the toy model of Gruzinov \& $\mathrm{Hu}$ (1998), in which the ionized regions are randomly distributed, is also shown. The heavy solid line shows the first-order anisotropy for $\Omega_{b}=0.02$ and $f_{\text {esc }}=1$.0. Note that only $C_{E l}$ contributes to the first-order effect, while in the second-order effect $C_{E l}$ and $C_{B l}$ are equal. 
the spheres remain ionized forever, the fractional ionization increases with increasing number density of ionized spheres during $\delta z$ until the universe is completely ionized. In Paper I, we chose $R=0.85 h^{-1} \mathrm{Mpc}, z_{i}=11$, and $\delta z=5$ in the Gruzinov \& $\mathrm{Hu}$ (1998) model to match the peak in the power spectrum of secondary temperature anisotropies predicted for our model $\mathrm{E}$. We choose the same parameters here and find that the toy model likewise matches the peak in the spectrum of polarization anisotropies for model E. For small $l$, little power is generated in the Gruzinov \& $\mathrm{Hu}$ (1998) model because, by design, the patches are uncorrelated.

To further clarify what determines the shape of the second-order anisotropy spectrum in our models, we carried out the following additional tests, shown in Figure 5. The first test was to force the ionized fraction $x_{e}$ to be uniform and equal to the same mean value as before, so that fluctuations $\delta_{e}$ in the free electron density are then simply equal to fluctuations $\delta$ in the total density. In this case, the angular power spectrum has an almost identical shape to models A-E, with amplitude about equal to that for models C, D, or E, but lower than that for models A or B (by factors of about 1.7 and 2.5 , respectively). In the next two tests (labeled "random" in Fig. 5), the total gas density was forced to be uniform (i.e., we set $\delta=0$ ), and put bubbles down at random positions, so that fluctuations in $\delta_{e}$ resulted only from the patchiness of the reionization. In one case, the bubble radii were chosen from the size distribution predicted by our galaxy formation model (this case is thus similar to model $\mathrm{D}$, except that model $\mathrm{D}$ had $\delta \neq 0$ ). In the other case, all bubbles were given the same comoving radius of $0.62 h^{-1} \mathrm{Mpc}$, which corresponds to the mean bubble radius (weighted by bubble volume) predicted by the galaxy model, at the redshift corresponding to the peak of the visibility curve. Both of these cases give power spectra with shapes (at large scales) similar to the analytical Gruzinov \& $\mathrm{Hu}$ (1998) model, and completely different from when fluctuations in $\delta$ are included. In the final test (labeled "clustered" in Fig. 5), we again forced the bubble radii to be equal at a given redshift, but placed them on random halos, and included the fluctuations in the total gas density. The starting value for the radii in this last case was again 0.62 $h^{-1} \mathrm{Mpc}$, but the spheres were then grown by a uniform factor at each redshift to produce the correct mean ionized fraction, as in model A. The power spectrum in this case is almost the same as in model $\mathrm{A}$, showing that the distribution in bubble sizes in the latter case does not have much effect.

From these tests, we conclude that in our models A-E, the shape of the power spectrum on scales large compared to the typical size of the ionized bubbles is determined primarily by the correlations in total gas density. However, in the case of patchy reionization, the amplitude depends on the spatial distribution of these patches, which produces biasing for the correlations in the ionized gas density relative to those in the total gas density, which in turn boosts the amplitude of the polarization fluctuations. For our models A-E, the boost in amplitude of the power spectrum, relative to the uniform $x_{e}$ case, varies from a factor of about $1-2.5$, the largest boost resulting from the case where the densest cells are ionized first (model B).

The amplitude of the second-order polarization power

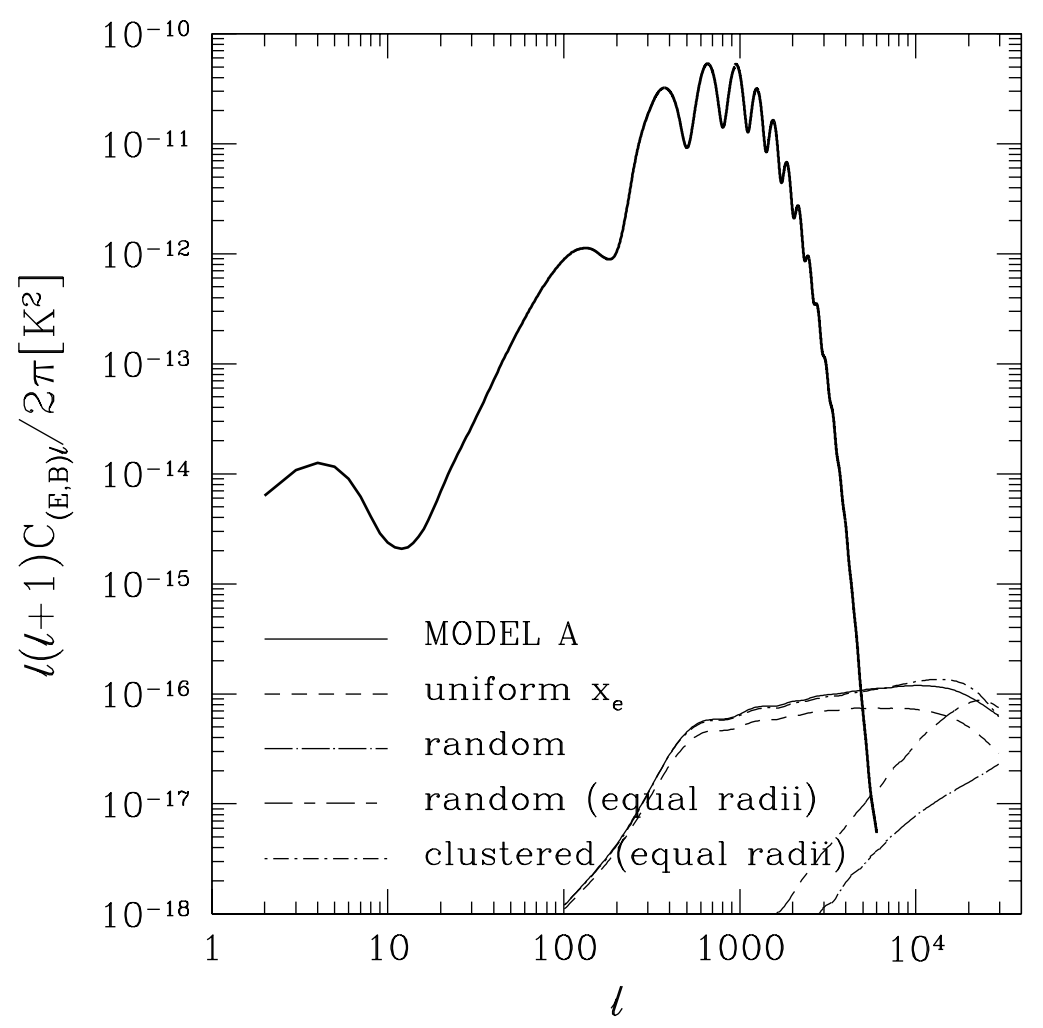

FIG. 5. - Effect on the second-order anisotropy of varying the assumed geometry of the reionized regions. The models are all computed for $\Omega_{b}=0.02$ and $f_{\text {esc }}=1.0$. The line for model A is repeated from Fig. 4. The line labeled "uniform $x_{e}$ " is for the case in which the ionized fraction is uniform and equal to the mean value in model A. For the line labeled "random" the total gas density is forced to be uniform, and spheres are put down at random positions but with the distribution of radii given by the galaxy formation model. The case "random (equal radii)" is the same, except that all spheres have the same comoving radius $\left(0.62 h^{-1} \mathrm{Mpc}\right)$. Finally, for the case "clustered (equal radii)," variations in the total gas density are included, but all spheres have equal radii at a given redshift and are placed on random dark halos. See the text for more details. 


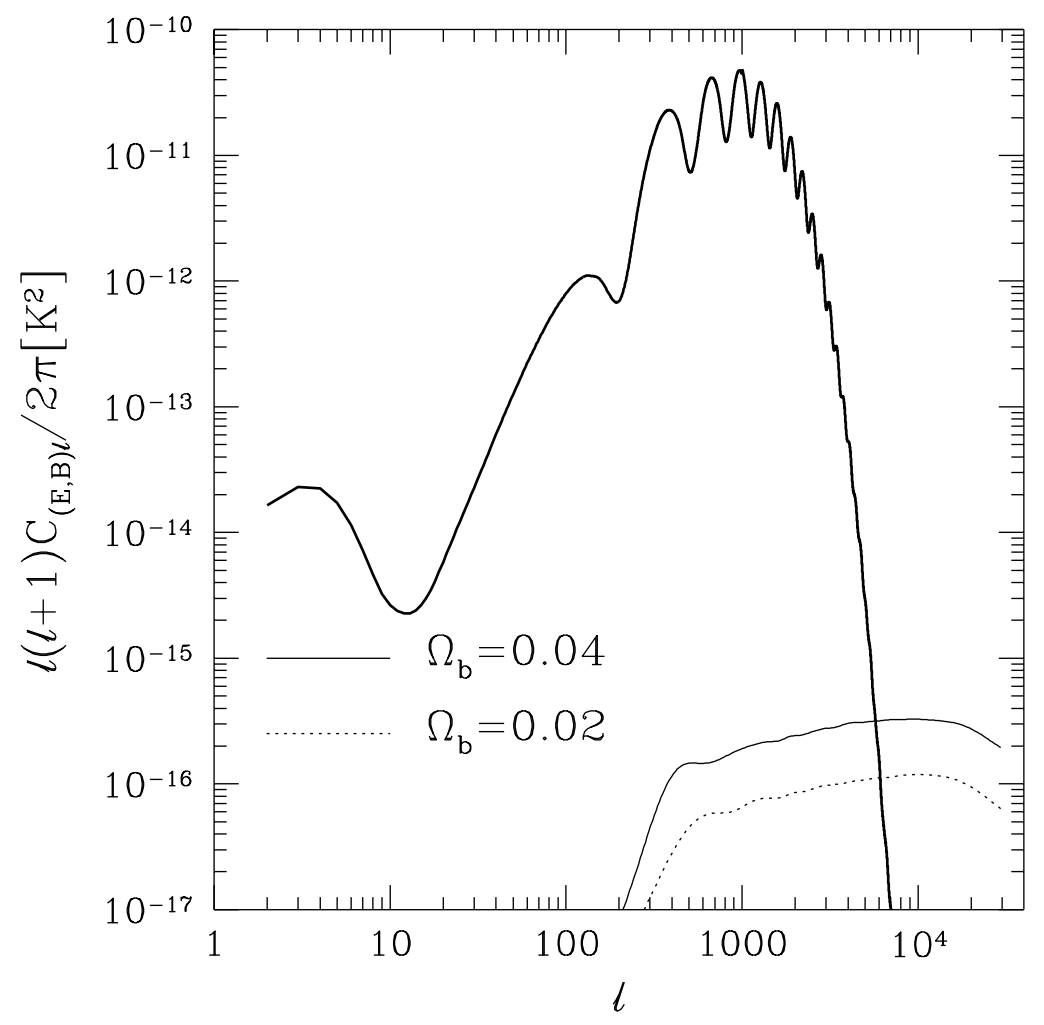

FIG. 6.-Effect on the secondary CMB polarization anisotropy spectrum of varying $\Omega_{b}$. The curves shown are all computed using model A. The solid line shows $\Omega_{b}=0.04$ while the dotted line shows $\Omega_{b}=0.02$. The heavy solid line shows the first-order anisotropy for the model $\Omega_{b}=0.04$ and $f_{\text {esc }}=1.0$. The power spectrum is multiplied by $T_{\mathrm{CMB}}^{2}$.

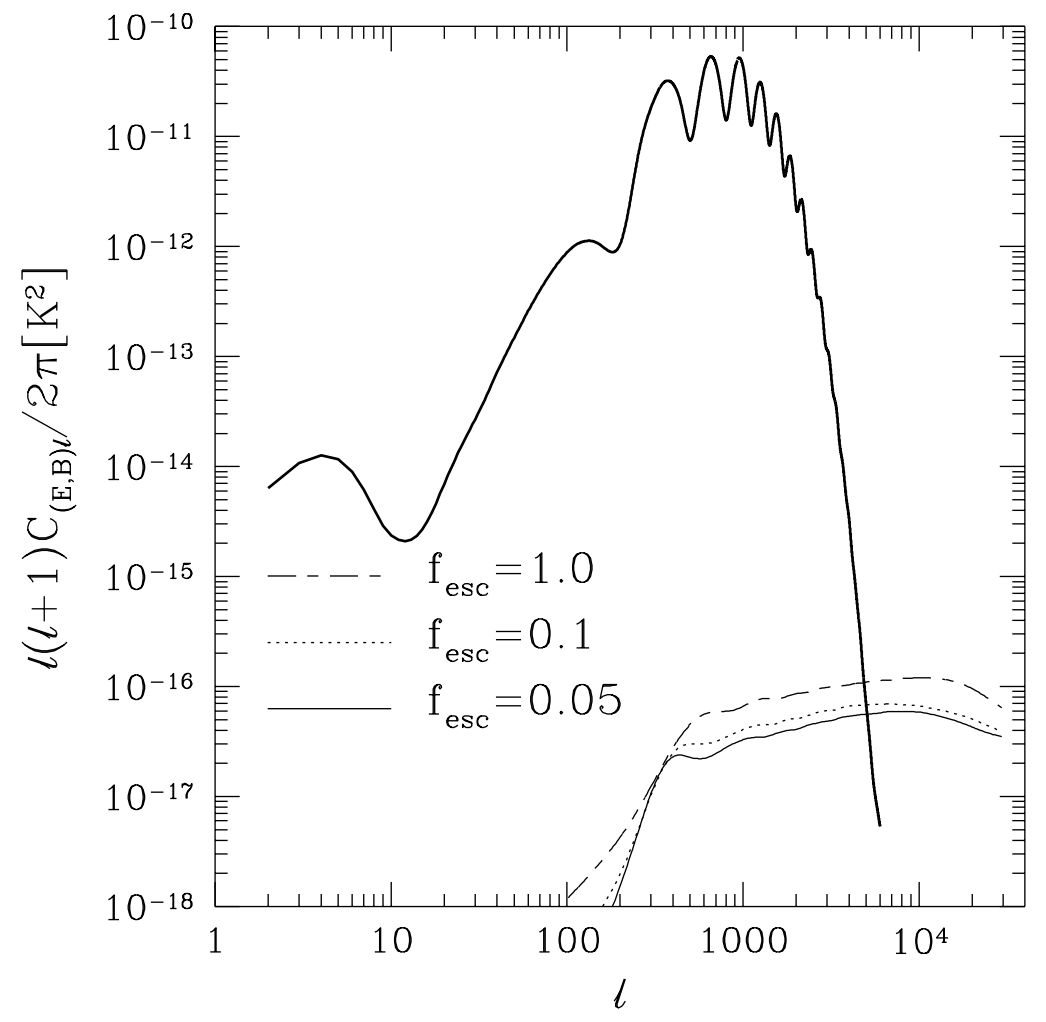

FIG. 7.-Effect on the secondary CMB polarization anisotropy spectrum of varying the escape fraction $f_{\text {esc }}$. The curves shown are all computed using model A and $\Omega_{b}=0.02$. Escape fractions of 1.0, 0.1, and 0.05 are shown as indicated in the figure. The heavy solid line shows the primary anisotropy for the model $\Omega_{b}=0.02$. The power spectrum is multiplied by $T_{\mathrm{CMB}}^{2}$. 
spectrum also depends on the value of $\Omega_{b}$, which affects the visibility function in equation (23). In our calculation, $\Omega_{b}$ is set to be 0.02 , which is lower than the current estimates based on Big Bang Nucleosynthesis which imply $\Omega_{b} h^{2}=$ $0.020 \pm 0.002$ (Burles, Nollett, \& Turner 2001). In Figure 6, we increase $\Omega_{b}$ to 0.04 (which results in $\kappa_{i}$ increasing to 0.048). If the evolution of the ionized regions were the same in both models, we would expect the amplitude to increase by a factor of 4 (eq. [14]). However, the delay of reionization resulting from this increase in $\Omega_{b}$, as was shown in Figure 1, reduces the factor to 2.8 .

So far we have set $f_{\text {esc }}=1.0$ only. Whilst this may in fact be a plausible value for high-redshift galaxies based on recent observations (Steidel, Pettini, \& Adelberger 2000), it is instructive to examine the effects of changing $f_{\text {esc }}$. Using model A we examine the effects on the second-order polarization anisotropies of varying $f_{\text {esc }}$. The results are shown in Figure 7. In contrast to the case of first-order anisotropies, the amplitude of the second-order anisotropies increases only weakly with increasing $f_{\text {esc }}$, with the extreme value $f_{\text {esc }}=1.0$ differing from the others by only a factor of about 1.6. This is because the source term for the first-order polarization anisotropies is the visibility function multiplied by the temperature quadrupole, $g(\tau) \Delta_{T 2}$. Since the temperature quadrupole changes slowly with time, we can say that the power spectrum is mainly determined by the visibility function. However, the source term for the second-order anisotropies is the visibility function multiplied by $\delta_{e}$, whose time evolution is roughly proportional to $1 /(1+z)$. Thus, the signal in the second-order anisotropies is more strongly weighted to low $z$, where the differences between models with different $f_{\text {esc }}$ are less significant.

We also plot the first-order power spectrum as heavy solid lines in Figures 4-7. This shows that the second-order signal dominates over the first-order signal on small scales $(l \gtrsim 6000)$. This will be useful for a high-resolution experiment since we will be able to distinguish the secondary signal from the primordial one.

\section{CONCLUSIONS}

We have investigated the polarization anisotropies created during the reionization epoch, for a realistic model of reionization by galaxies, in particular focusing on the second-order effect. It is found that the boost in the largescale polarization anisotropy from the first-order effect is very sensitive to the optical depth due to reionization, with larger optical depths giving larger boosts. Using a semianalytic model of galaxy formation to calculate reionization (Paper I), the optical depth from the present to the recombination epoch, $\kappa_{i}$, is predicted to be in the range 0.014-0.048, for $\Omega_{b}$ in the range $0.02-0.04$, and the escape fraction of ionizing photons from galaxies $f_{\text {esc }}$ in the range 0.05-1.0. This first-order effect causes a new peak in the polarization at large scales with amplitude around $0.05-0.15 \mu \mathrm{K}$. The position of the peak is determined by the size of the horizon at reionization. If this peak can be detected by future experiments such as Planck, it would offer valuable evidence to guide our understanding of reionization of the universe.

We have also studied the second-order polarization effects resulting from the coupling of fluctuations in the free electron number density, $\delta_{e}$, with the quadrupole of the temperature anisotropies. We obtained $\delta_{e}(x, t)$ by combining the semianalytic model of galaxy formation with a highresolution $N$-body simulation, as in our previous work (Paper I). The semianalytic model determines the average ionized fraction at each redshift, but the spatial distribution of ionized gas is based on using the simulation to determine the locations of the ionizing sources and density field of the IGM. We determine which regions of the IGM are ionized using one of five toy models. We summarize our results for the second-order polarization anisotropies as follows: (1) The second-order effect dominates over the first-order effect on small scales $(l>6000)$. (2) The $B$-mode of polarization is induced as well as the $E$-mode. The angular power spectra of these two modes $C_{E l}$ and $C_{B l}$ are the same. (3) The shapes of the $C_{(E, B) l}$ are very similar in all of the toy models we considered for the spatial distribution of the ionized regions, but differ considerably from the toy model of Gruzinov \& $\mathrm{Hu}$ (1998), having much more power on larger angular scales. The reason for this difference is the spatial correlations of the total gas density in our model, which produce corresponding large-scale correlations in the density of ionized gas also. The shape of $C_{(E, B) l}$ on large scales is determined mostly by the correlations in the total gas density, but its amplitude is sensitive to the geometry of the ionized regions, which determines the biasing factor of the correlations in ionized density relative to those of the total gas density. (4) The difference in amplitude between our different toy models for the spatial distribution of ionization is large in the second-order effect, for example, models $\mathrm{B}$ and $\mathrm{C}$ differ by a factor of 2.5 . Thus, they provide a very important constraint on galaxy formation and reionization, in spite of the fact that the shapes of all curves are similar. The cosmological parameters which also affect the amplitude of the second-order effect will be determined by forthcoming precise measurements of CMB anisotropies by $M A P$ and Planck. Therefore, we expect that when the second-order polarization is observed, its amplitude will provide an important signature of the galaxy formation and reionization processes. (5) The amplitude also depends on the value of $\Omega_{b}$. If the evolution of the ionized regions were the same for any value of $\Omega_{b}$, we would expect the amplitude to be proportional to $\Omega_{b}^{2}$. However, the scaling in our model is somewhat weaker than this, because increasing the value of $\Omega_{b}$ delays reionization. In our calculation, the secondorder power spectrum for an $\Omega_{b}=0.04$ model has an amplitude 2.8 times greater than for an $\Omega_{b}=0.02$ model. (6) The amplitude of $C_{(E, B) l}$ depends only weakly on $f_{\text {esc }}$ (and so on the redshift of reionization). Since the amplitude of the power spectrum for the extreme value of $f_{\text {esc }}=1.0$ differs from that with $f_{\text {esc }}=0.1$ only by a factor of about 1.5 , the results in Figures 4 and 6 should be only weakly dependent on the true value of $f_{\text {esc }}$.

While second-order effects can make a significant contribution to the temperature anisotropy $(\sim 0.1-1 \mu \mathrm{K})$, the second-order polarization anisotropies generated by inhomogeneous reionization are, as expected, very small. We conclude that the signals are typically of order $10 \mathrm{nK}$ on arcminute scales, comparable to estimates from other authors studying Vishniac-type polarization (Seshadri \& Subramanian 1998) or toy models of patchy reionization (Weller 1999). A signal of this amplitude is below the detectability limits of the Planck Surveyor mission, which is the most accurate experiment in the near future. However, it is worthwhile to note that this second-order effect generates the same power in the $E$ - and $B$-modes. Thus, a detection of $B$-mode polarization on arcminute scales or below is essential to distinguish the second-order effect from other possible effects. Since it provides more information about galaxy formation and the process of reionization than the 
first-order effect, detection of this signal should be a key aim of a post-Planck experiment with increased sensitivity and resolution in the next decade. Among currently planned instruments, the most useful one for detecting the secondorder signal is probably the millimeter-wavelength interferometer Atacama Large Millimeter Array, which will be able to measure CMB fluctuations on arcminute scales. Since the second-order signal is very small, polarized galactic foregrounds, from dust (e.g., Prunet et al. 1998) and synchrotron emission (e.g., Baccigalupi et al. 2001), are a concern. However, the amplitude of these foregrounds on arcminute scales is currently unknown, as observational estimates have been obtained only for larger scales $(l \lesssim 100-1000)$. The detectability of the CMB polarization signal can thus only be decided by future observational work.
We thank U. Seljak and M. Zaldarriaga for the use of CMBFAST; the Virgo Consortium for making available the GIF $N$-body simulations used here; and Shaun Cole, Carlos Frenk, and Carlton Baugh for allowing us to use their model of galaxy formation. G. C. L. thanks N. Seto for useful discussion and acknowledges the fellowship of Interchange Association. N. S., A. N., and C. G. L. acknowledge the kind hospitality of Carlos Frenk and the physics department of University of Durham during the TMR network meeting. N. S. is supported by the Sumitomo Foundation. C. G. L. acknowledges support at SISSA from COFIN funds from MURST and from ASI. A. N. and A. J. B. acknowledge the support of the EC RTN network "The Physics of the Intergalactic Medium." A. N. was supported by a grant from the Israeli Academy of Science.

Baccigalupi, C., et al. 2001, A\&A, 372, 8

Benson, A. J., Nusser, A., Sugiyama, N., \& Lacey, C. G. 2001, MNRAS, REFERENCES 320, 153 (Paper I)

Bond, J. R., \& Efstathiou, G. 1984, ApJ, 285, L45

Bruscoli, M., Ferrara, A., Fabbri, R., \& Ciardi, B. 2000, MNRAS, 318, 1068

Burles, S., Nollett, K. M., \& Turner, M. S. 2001, ApJ, 552, L1

Cole, S., Lacey, C. G., Baugh, C. M., \& Frenk, C. S. 2000, MNRAS, 319, 168

Gnedin, N. Y. 2000, ApJ, 535, 530

Gnedin, N. Y., \& Jaffe, A. H. 2001, ApJ, 551, 3

Gruzinov, A., \& Hu, W. 1998, ApJ, 508, 435

Hu, W. 2000, ApJ, 529, 12

Jaffe, A. H., \& Kamionkowski, M. 1998, Phys. Rev. D, 58, 043001

Kaiser, N. 1984, ApJ, 282, 374

Ma, C.-P., \& Bertschinger, E. 1995, ApJ, 455, 7

Miralda-Escudé, J., Haehnelt, M., \& Rees, M. J. 2000, ApJ, 530, 1

Newman, E., \& Penrose, R. 1966, J. Math. Phys., 7, 863

Ng, K.-W., \& Liu, G.-C. 1999, Int. J. Mod. Phys., D8, 61

Ng, K. L., \& Ng, K.-W. 1996, ApJ, 456, 413

Peebles, P. J. E., \& Yu, J. T. 1970, ApJ, 162, 815

Prunet, S., Sethi, S. K., Bouchet, F. R., \& Miville-Deschenes, M.-A. 1998, A\&A, 339, 187

Sakurai, J. J. 1985, Modern Quantum Mechanics (New York: AddisonWesley)

Sachs, R. K., \& Wolfe, A. M. 1967, ApJ, 147, 73

Sato, H. 1994, Prog. Theor. Phys., 92, 37

Seljak, U., \& Zaldarriaga, M. 1996, ApJ, 469, 437

Seshadri, T. R., \& Subramanian, K. 1998, Phys. Rev. D, 58, 063002

Shapiro, P. R., \& Giroux, M. L. 1987, ApJ, 321, L107

Smoot, G. F., et al. 1992, ApJ, 368, L1

Springel, V., White, M., \& Hernquist, L. 2000, ApJ, in press

Steidel, C. C., Pettini, M., \& Adelberger, K. L. 2001, ApJ, 546, 665

Sunyaev, R. A., \& Zel'dovich, Ya. B. 1980, MNRAS, 190, 413

Valageas, P., Balbi, A.. \& Silk, J. 2001, A\&A, 367, 1

Vishniac, E. T. 1987, ApJ, 322, 597

Weller, J. 1999, ApJ, 527, L1

Zaldarriaga, M. 1997, Phys. Rev. D, 55, 1822

Zaldarriaga, M., \& Seljak, U. 1997, Phys. Rev. D, 55, 1830

- 1998, Phys. Rev. D, 58, 023003 